Action-specific effects in perception and their potential applications:

\title{
A reply to commentaries
}

Word Count: 4353

(C) 2016. This manuscript version is made available under the Elsevier user license http://www.elsevier.com/open-access/userlicense/1.0/ 
According to the action-specific approach to perception, people perceive the spatial layout of the environment relative to their ability to act in this environment. Hills appear steeper and distances appear farther to perceivers who would have to exert more effort to traverse the space. Objects that are more difficult to reach and grasp appear farther and bigger compared with objects that are easier to reach and grasp. In our target article (Witt, Linkenauger, \& Wickens, 2016), we provided a brief overview of these action-specific effects, and then proposed ways in which considering perception as being action-specific could be applied to safety, rehabilitation, diagnostics, and communication. Scholars authoring two of the commentaries commended this extension and called for research to test these speculations (Balcetis \& Cole, 2016; Eves, 2016). In contrast, the two other commentators argued these speculations are premature given the outstanding issues related to the theoretical claims of the action-specific effect (Gray, 2016; Loomis, 2016). Our response starts with these theoretical issues.

\section{Action-Specific Approach to Perception}

The action-specific approach currently provides a broad framework for describing ways that a person's ability to act influences spatial perception. To date, much of the research has focused on documenting the phenomenon (including the conditions under which action-specific effects occur) and addressing the issue of cognitive influences on perceptual judgments.

\section{Defining Action}

With the discovery of any new phenomenon, much research is dedicated to understanding the conditions under which the effects do and do not present themselves. Yet Loomis (2016) criticized the evolving view of action-specific effects that results from these new discoveries. To some extent, Loomis's concern is well-founded given that lack of clear definitions contributed to the demise of the New Look approach to perception (Carter \& Schooler, 1949). Yet, the more we investigate action- 
specific effects, the more we learn, and for this reason, our approach is updated. The point of science is to refine and develop theories by conducting research. Loomis's complaint of a constantly moving target is only problematic if one's sole goal is to strike down the approach, rather than engage in the process of discovering more about our perceptual systems.

Allowing new evidence to update the approach is part of the pathway to attaining a precise definition of action. By criticizing the approach for having not achieved this goal already, Loomis (2016) undermines the difficulty of declaring this definition by ignoring all the nuanced ways that action can impact spatial perception. As Eves (2016) argued in his commentary, even one aspect of action-specific effects (effort) is likely to involve multivariate factors such as weight, fatigue, fitness, sex, and age. One of us (Witt) proposed a 4-factor definition for which action consists of the likelihood of success of a given action, the energetic costs associated with attempting the action, the benefits gained from successfully performing the action, and the costs associated with failing to perform the action (Witt \& Sugovic, 2013b). Action has not been simply defined because action's influence on perception is not simple.

In contrast, Balcetis and Cole (2016) argued for a simpler definition by claiming that all actionspecific effects can be reduced to understanding how motivation impacts spatial perception. They claimed that all effects can be understood within an approach versus avoidance framework. It is not clear to us how a motivational framework can explain many action-specific effects such as that of athletic success on apparent size of the target (e.g. Witt \& Proffitt, 2005). Presumably all of the softball players were motivated to hit the ball, but those who had more success judged the ball as bigger compared with those who had less success. The simplistic approach/avoidance framework does not even explain all effects of motivation, as desirable objects and fearful objects both appear closer despite one prompting approach and the other prompting avoidance (Balcetis \& Dunning, 2010; Cole, Balcetis, \& Dunning, 2013). This is not to say that motivation is irrelevant, but to our minds, the effect of 
motivation is separate from that of action. This stance is supported by research showing separate and independent effects for action and motivation (Witt \& Sugovic, 2013b). In a series of studies, participants attempted to block a threatening object (a spider) or a neutral object (a ball) with various sized paddles. Two parallel effects emerged, one for the object and one for the paddle size. Spiders were judged as faster than balls, and both objects were judged as faster when the paddle was small than when it was big. The lack of significant interaction indicates the two effects are parallel and separate. Thus, when both performance and motivation are included within the same study, the effect of action cannot be reduced to mere motivation, and vice versa; both produce their own effects.

We reject the claim that all action-specific effects can be considered effects of approach versus avoidance, and we also reject the idea that action can be reduced to a simplistic explanation. Action's effects on spatial perception are varied and nuanced. Thus, we agree with Eves's (2016) call for larger studies that can simultaneously measure multiple aspects of action and with Loomis's implicit call for studies that involve parametric variation of action. Appreciating the varied ways that action can impact spatial judgments is also especially important because the various aspects of action may impact perception via different mechanisms (Proffitt \& Linkenauger, 2013; Witt \& Riley, 2014). If action is as intricately linked to spatial perception as we have argued, there are likely to be multiple ways in which the two are connected, rather than via a single mechanism. We return to the issue of mechanisms later. Embedded in our approach is that action is selected by the perceiver's intention. Eves (2016) questioned the need for including intention in the approach as much of his (and other's) research reveal action-specific effects without appealing to intention. As he pointed out, none of the studies on hill slant perception required participants to ascend the hill, and in the studies on stair slant perception, in many cases, participants had already descended the stairs and were walking away when asked to estimate slant. He further points out that he has obtained similar effects when asking perceivers to judge slant when viewing images of stair cases, in which case, there is certainly no intention to walk up 
the picture. Eves may very well be right. However, we are unwilling to let go of intention as a critical component of selecting action at this time. It is not unreasonable to presume that the ground plane solicits a default and implicit intention to walk, and so hills and stairs are viewed with respect to walking even if no explicit task is set before the viewers. With respect to images, the perceptual system is likely to use the very same processes as used to view a real scene. This is why effective images, drawings, and paintings are ones that recreate the same kind of perspective cues as the real world. If the visual system had a whole different set of rules and processes for images, images could look a lot different. Consequently, evidence that action-specific effects can be observed when viewing life-sized images does not necessarily rule out intention as a relevant factor. Nevertheless, our claims regarding intention are certainly speculative and need to be empirically examined.

\section{Accounting for Cognitive Bias}

Critics of the action-specific account are primarily concerned with the issue of cognitive bias (also known as demand characteristics, task demands, and response bias). Many proponents of the action-specific approach have shown an appreciation for the nuanced ways that cognitive biases may account for purported action-specific effects. We have conducted dozens of studies specifically on this issue, and used many strategies that have been proven to be effective in the literature and specifically suggested by critics of this approach, such as the use of indirect measures and action-based measures.

In the current article, we take the strong stance that action-specific effects can be perceptual.

This is not, as Loomis (2016) claims, throwing caution to the wind. This is the direct result of a decade of research on tackling this particular issue. We have used several indirect methodologies, including, but not limited to, perceived aspect ratio (Linkenauger, Leyrer, Buelthoff, \& Mohler, 2013), perceived parallelism (Witt, 2011), perceived shape (Witt, 2011), weight perception (Linkenauger, Mohler, \& Proffitt, 2011), and affordance judgements (Linkenauger et al., 2014), and several action-based 
measures such as blindwalking (Stefanucci \& Proffitt, 2009; Tenhundfeld \& Witt, in preparation; Witt, Proffitt, \& Epstein, 2010) and reaction time (Witt \& Sugovic, 2013a). Having conducted the very types of experiments suggested by Loomis and others, and having found support that the effects are perceptual, we are justified to refer to action-specific effects as perceptual.

One reason for the discrepancy between our strong claim that these effects can be perceptual and Loomis's (2016) concern that the evidence is not sufficient to make such a claim is that there is no standard set in the field as to what evidence is needed to assert an effect is perceptual. Loomis himself does not indicate what evidence would be sufficient, only that the current evidence is not. Critics have created a moving target themselves by calling for indirect and action-based measures, and then minimizing the outcomes found with these measures. Give us a standard, and let us use the standard to evaluate the nature of action-specific effects. Testing for perceptual effects should be an empirical question, not one dependent on "paying close attention to [one's] own perceptual experience" (Loomis, 2016, p. X).

\section{Phenomenology}

Although this has not been explicitly stated, we speculate that one of the reasons why critics hold on so dearly to the cognitive bias explanation is that it provides them a theoretical outlet to reject claims of perceptual effects, and this rejection is actually driven, as Loomis (2016) admits, by their lack of phenomenological experience with action-specific effects. This is understandable. People want to believe their own eyes, and so if their eyes do not reveal an effect to them, it is hard to believe in the effect. Gray (2016) also asserts that action-specific effects are not noticeable.

What does it mean for an effect to be noticeable? Neither Loomis nor Gray provide definitions. In his example about his student, Loomis implies that being noticeable means that one can experience a difference between one's current perception of an object and one's memory for that object. That his 
student did not notice effects of fatigue suggests that his perception of hill slant when he was fatigued was not noticeably different than his memory for the hill slant when he was rested. This student's experience is not shared by all. Many athletes have been heard exclaiming that when they are in the zone, baseballs appear bigger or tennis balls appear slower. Evidently, the anecdotal evidence from athletes and scientists is mixed. However, one study empirically measured the noticeability of actionspecific effects in track runners (Witt, 2015). The track has the advantage that it is well marked, so runners can compare their experience of the track to knowledge about the actual distances. This allows runners to experience a discrepancy between what they see and what they know. In the study, over $80 \%$ of track runners expressed that the track appeared longer when they were fatigued (see Figure 1). Other studies have noted that participants spontaneously reported noticing changes in their perception when large manipulations of the body are employed by using virtual reality and magnifying lenses (Linkenauger, Bulthoff, \& Mohler, 2015; Linkenauger et al., 2013; Linkenauger, Ramenzoni, \& Proffitt, 2010). Thus, action-specific effects may very well be noticeable. 


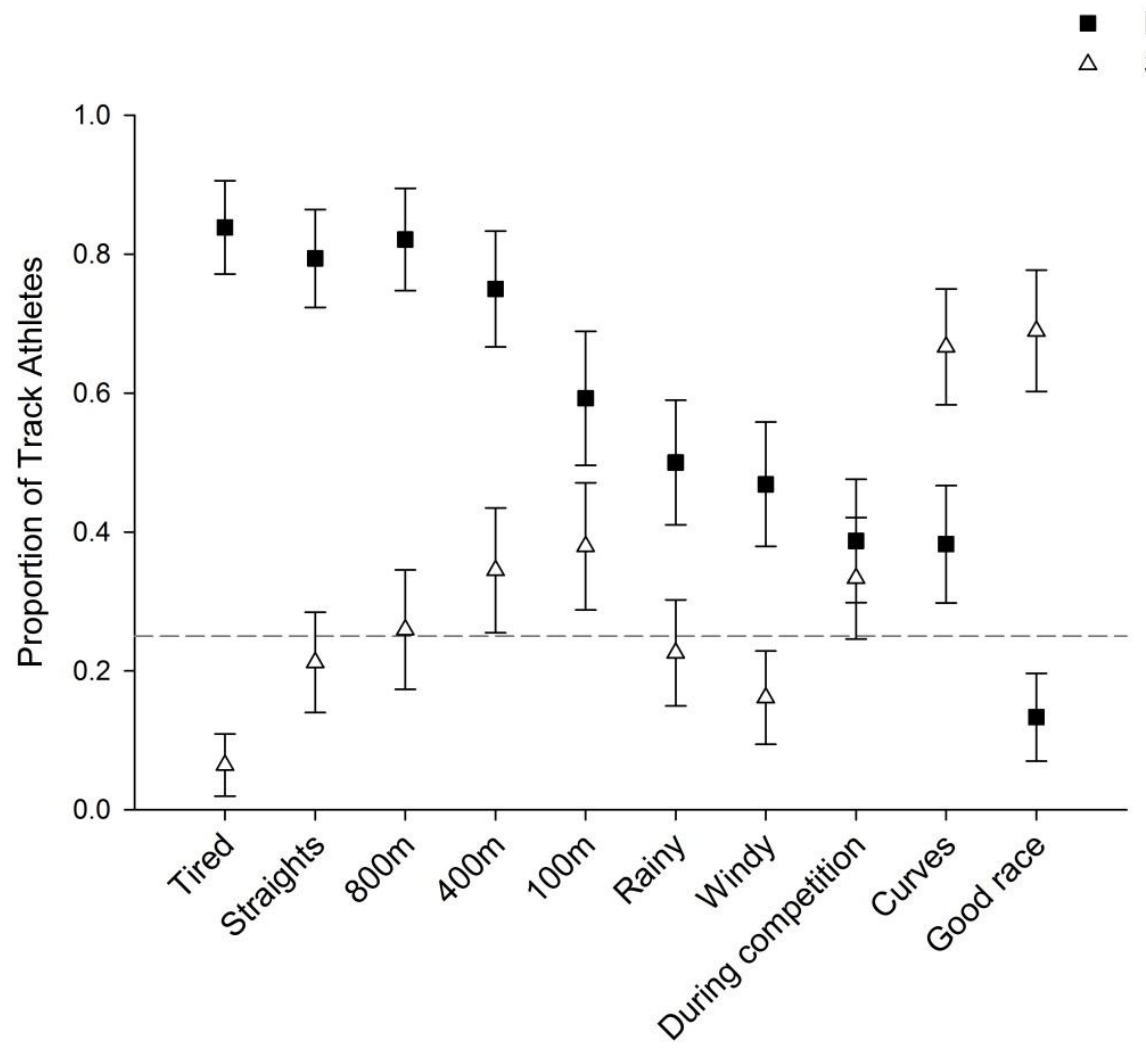

Figure 1. Proportion of surveyed track athletes who reported that a track appeared longer or shorter as a function of the part of the track ("straights" vs. "curves"), the length of the race, and environmental or performance-related conditions. "Good race" refers to whether the athletes felt they were having a good race day. Error bars are 1 SEM, calculated within-subjects. Data are from Witt (2015). Dotted line is at 25\%, which corresponds to previous levels of documented awareness (Durgin et al., 2009).

A second definition of being noticeable, which was alluded to by Gray's context of visual illusions, suggests that being noticeable means seeing a difference between two objects, even though the two objects are the same. For instance, the Ebbinghaus illusion is noticeable to most people because they experience a difference in the size of the center circles. With this definition of being noticeable, action-specific effects can also be noticeable when assessed with a visual matching task. For example, in a virtual environment, participants estimated the length relative to the width of a block and judged whether or not the block could be grasped with their virtually-rendered hands (Linkenauger et al., 2013). Graspable blocks with equal widths and lengths (i.e. squares) appeared to have different 
length sizes when the perceiver imagined grasping them with a larger virtual hand than with a smaller virtual hand. In other words, the two sides looked noticeably different, even though they were the same. As another example, targets on a hill are verbally judged to be farther away, presumably because walking to them would require more effort than walking on flat ground (Stefanucci, Proffitt, Banton, \& Epstein, 2005). In follow-up experiments, participants were asked to instruct an experimenter to move closer or farther away until the distance to the experimenter was the same as the distance to a target presented up a hill. When the experimenter was at the same distance as the target, participants instructed the experimenter to move farther away, suggesting the two distances did not appear the same even though they were the same (Tenhundfeld \& Witt, in preparation). These experiments show that action-specific effects can be noticeable if noticeability is defined as seeing two objects as being different even when they are the same.

Loomis, and others (Firestone \& Scholl, in press), call for amazing perceptual demonstrations. Action-specific demonstrations cannot be uploaded to a webpage like other kinds of perceptual phenomena because these effects are not just about optical cues but also about action. The perceiver must have the potential to act. This lack of sensitivity to action in research on perception is likely why these effects have only been recently discovered. To demonstrate an action-specific effect, consider what kinds of perceptual demonstrations are available for other kinds of perceptual phenomenon in the real world, such as egocentric distance compression. Loomis (2016) does not indicate how he can phenomenally experience egocentric distance compression, but presumably demonstrations in these cases require visual matching tasks. In this case, people are encouraged to perform tasks such as those described in the previous paragraph in order to experience action-specific effects for themselves. 


\section{Determining the Underlying Mechanism}

Although proponents of the action-specific approach generally agree about the main claim that a person's ability to act influences spatial perception, there is little agreement on the mechanism underlying this effect, and even less evidence. As Gray (2016) indicates, this is a big gaping hole in the field that needs to be filled, a point to which we fully agree. Balcetis and Cole (2016) argue that motivation provides the mechanism, but as noted earlier, motivation cannot explain all action-specific effects. Furthermore, no description is offered of how motivation is the mechanism in the sense of explaining how motivation interacts with optical cues.

Some researchers have proposed that action's effect on spatial perception is indirect and operates via attention. This hypothesis is supported by studies showing a relationship between eye movements, performance, and perceived size (Gray, Navia, \& Allsop, 2014), but this notion is countered by other evidence showing no relationship between eye movements and action's effect on estimated size (Van der Hoort \& Ehrsson, 2014) and speed (Witt, Sugovic, \& Dodd, in press). Others have likened action-specific influences as being similar to cross-modal effects (Witt, 2015; Witt \& Riley, 2014), but there has not yet been any empirical research to evaluate this proposal. A third proposal, called the perceptual ruler account, is that action-specific effects are like other types of body-based scaling effects (Proffitt \& Linkenauger, 2013). An example of a body-based scaling effect is the use of eye height for perceiving distance and object height (Sedgwick, 1986). The perceptual ruler account suggests that other aspects of the body are used to scale all optical angles. Hand size can be used to scale the size of graspable objects, and performance variability can be used to scale the size of hittable targets.

All three of these proposed mechanisms are consistent with multiple approaches to vision, including the approach emphasized by Loomis (see Philbeck \& Witt, 2015) and a modified version of Gibson's (1979) theory of direct perception (see Witt \& Riley, 2014). However, action-specific effects are also consistent with an emerging view of perception as being a phenotypic expression (Proffitt \& 
Linkenauger, 2013). In this view, perception is not about creating perceptual representations, but rather evolved to support the needs and goals of the organism, and consequently, percepts of spatial layout are scaled to the morphology, physiology, and behavioral repertoire of the organism.

Loomis comes from a long tradition of assuming that the purpose of perception is to create an isomorphic representation of the physical environment (isomorphic meaning that they are similar in form and relations). Much of the empirical work underlying this perspective is concerned with "the mapping between physical and visual space" and "the intrinsic geometry of visual space" (Loomis, 2016, pg. X). It is easy to believe that what people perceive is actually what is out there (or a faithful representation of that), yet this is not the purpose of perception. Creating an isomorphic representation of the environment would actually be detrimental to the organism as it requires more energy to create and sustain than it provides (see Singh \& Hoffman, 2013). Physical accuracy (or isomorphism) is not analogous to adaptiveness. Instead, the perceptual system processes information about the environment that signifies something relevant to the perceiver. Action-specific effects are consistent with this view, because they reveal that people see the environment as it relates to their own abilities; they see it with respect to its relevance to their own potential for action.

Physical space can be infinitely small (molecules) or infinitely big (galaxies). Consequently, space in terms of the world of physics provides perceivers nothing on which to ground their percepts. Perception has to be relative to the individual. The ground is only a solid surface, because it is denser than one's body. Additionally, people only perceive objects of a size that are relevant to their action capabilities. People cannot see an individual molecule no more than they can grasp it. In contrast to Loomis's representational perspective, the action specific perspective grounds the perception of spatial layout in the phenotype of the individual, thereby avoiding theoretical problems associated with traditional representational accounts (see Proffitt \& Linkenauger, 2013). Put simply, there is no concrete physical environment to perceive that can be divorced from the perceiver, and the 
environment that is perceived is tailored to the individual. Consequently, the action-specific approach is more rooted in evolution because of its emphasis on the individual and her ability to act compared with traditional theories.

\section{Applications of the Action-Specific Approach to Perception}

For action-specific effects to be relevant for applications such as safety and rehabilitation, they must affect perception, and the kinds of effects that they produce in perception must be sufficient to bring about change in behavioral decision or performance. Section 1.4 of our target article was dedicated to building a case that action-specific effects can be perceptual. In Section 2.0, we illustrated how perceptual effects can impact action. Our examples were never intended to be of action-specific effects (as thought by Loomis and Gray), but rather our examples were of the kinds of changes that are similar in outcome (but not in mechanism) to action-specific effects. We were illustrating how changes in perception (in general) can impact behavioral performance and decisions. Such examples set the stage for action-specific effects having their own impact on action.

Illustrating that differences in perception can have an impact on action may seem trivial given that perception obviously drives action. Yet, both Gray (2016) and Eves (2016) questioned this point. Gray stated that it is less plausible that perceivers have "such a finely tuned mechanism" (2016, p. X), and Eves stated that people were "unlikely to be carefully considering their perceptual options" (2016, p. X). What both of these authors are ignoring is a wealth of research on affordance perception, which demonstrates that perception is finely tuned. For example, a difference of only 5 inches in stair height can render a step from being climb-on-able to being too tall to climb (Warren, 1984). With respect to careful consideration, we (and others) argue that perception takes care of the careful consideration for the perceiver, so the perceiver only has to look and see what is and is not possible. Perception takes the thinking out of the process of seeing affordances, or possibilities for action. This means that perceivers 
are not restricted to making solely "binary decisions (e.g. avoid climbing all stairs)" (Gray, 2016, p. X), but rather can easily and directly see what actions are available to them as well as their potential consequences, which is a much more plausible and evolutionary adaptive mechanism.

Although the affordance perception literature demonstrates that perception is finely tuned to action boundaries, we agree with the commentators that it still needs to be explored whether actionspecific effect sizes are sufficiently large to be practically relevant. A purpose of our target article is to raise the call for this kind of research, as the topic has been largely neglected. One exception is work by Gray, who mimicked the action-specific effects on perceived ball size and demonstrated that these differences were sufficient to impact batting decisions (Gray, 2013). Another exception is work by Eves (Eves, Thorpe, Lewis, \& Taylor-Covill, 2014), who showed that people who see stairs as steeper are more likely to take the escalator. More work is needed to determine if effect sizes in other action-specific domains are also sufficient to produce changes. The $2 \mathrm{~mm}$ effect in the archery study, which measured proximal size, and at $50 \mathrm{~m}$, a $2 \mathrm{~mm}$ proximal effect is actually quite large, is therefore likely sufficient (Lee, Lee, Carello, \& Turvey, 2012). But is a $5 \mathrm{~cm} / \mathrm{s}$ effect in the Pong studies (e.g. Witt \& Sugovic, 2012) sufficient to produce changes in behavioral decisions in, for example, tennis or hunting? That remains to be determined.

We are not suggesting that action-specific strategies for altering perception replace other strategies such as the use of visual illusions. As both Gray (2016) and Eves (2016) pointed out, actionspecific biases are tailored to the individual perceiver, which raises the issue of how to alter the environment in a way that is adaptive given these differences amongst individuals. Here, we urge designers to consider not all perceivers but the action capabilities of the individuals most likely to use that space. For example, senior centers and assisted living facilities both cater to perceivers with reduced abilities (and corresponding differences in perception; Bian \& Andersen, 2013; Sugovic \& Witt, 2013). Certain gyms and trainers cater to people who weigh more than others, and they should be 
aware of perceptual differences within their clients (Sugovic, Turk, \& Witt, in press; Taylor-Covill \& Eves, in press). In these cases, action-specific effects that are tailored to individuals can be useful.

\section{Rehabilitation and Diagnostic Measures}

Loomis (2016) distinguishes body-based effects from action-specific effects, but we consider body-based scaling to be a subset of action-specific effects (Proffitt \& Linkenauger, 2013). Several studies have shown that body morphology influences perceived extents (Leyrer, Linkenauger, Bulthoff, \& Mohler, 2015; Linkenauger et al., 2015; Sugovic et al., in press; Taylor-Covill \& Eves, in press; Van der Hoort \& Ehrsson, 2014). Neglect in near space in amputees is likely indicative of this phenomenon, and research on tool use in patients with neglect speak to this idea of expansion of near space as having an effect on neglect (e.g. Berti \& Frassinetti, 2000), as we suggested.

Measures of improved behavioural ability and fluency are available for prosthetic limbs, but they are not perfect (Datta, Selvarajah, \& Davey, 2004; Dudkiewicz, Gabrielov, Seiv-Ner, Zelig, \& Heim, 2004; Lindner, Nätterlund, \& Hermansson, 2010; Roeschlein \& Domholdt, 1989; Wright, Hubbard, Jutai, \& Naumann, 2001). This is one of the reasons that diagnostic practitioners employ multiple measures. The efficacy of measures of spatial perception is that they do not rely on verbal reports of the prosthetic but still capture the patients' perceptions of the prosthetic's usefulness (actual usefulness in behavioral measures may differ from the patients' actual expectations of their abilities, this is indeed true for handedness, see Linkenauger, Witt, Bakdash, Stefanucci, \& Proffitt, 2009), and thereby, spatial judgements may have some predictive power beyond the currently used measures. However, we cannot be sure until we test this presumption empirically. This possible utility of spatial perception as a diagnostic tool for prosthetics is similar to what we encountered when testing individuals with chronic pain (Witt et al., 2009). The motivation of the anaesthetist with whom we collaborated (Dr. Cook) was 
the development of an objective measure of chronic pain, because current diagnostic measures were woefully inadequate.

\section{Summary}

In conclusion, there is a plethora of research on the perceptual nature of action-specific effects.

This research has used the standard strategies to assess whether an effect is perceptual such as indirect and action-based measures, and the data support a perceptual account in several cases (e.g. Witt, Sugovic, Tenhundfeld, \& King, in press). In conducting careful experiments that take into account nuanced effects of cognitive bias and varied effects of action, we have also determined a number of boundary conditions. As a consequence of the results, the action-specific approach as evolved by both expanding relevant aspects of action (such as driving in a car; Moeller, Zoppke, \& Frings, in press) and restricting other sorts of top-down effects (such as beliefs about body size; Sugovic et al., in press). One might characterize these advancements as a moving target that knows no bounds. We prefer to let empirical data dictate theory, rather than ignore empirical results that contradict one's own personal perceptual experiences.

Here, we also call for more research on extending action-specific effects to human factors and applications. We have argued for the feasibility of applications for action-specific effects, but again, empirical data are needed. Researchers should not wait to extend action-specific effects beyond the laboratory. Although the conversation regarding the perceptual nature of action-specific effects is likely to continue, research on applications can contribute to this conversation. 


\section{References}

Balcetis, E., \& Cole, S. (2016). Motivating the action-in-perception hypothesis: Approach and avoidance motives as a process-model for action's effects on perception. Journal of Applied Research in Memory and Cognition.

Balcetis, E., \& Dunning, D. (2010). Wishful seeing: more desired objects are seen as closer. Psychological Science, 21(1), 147-152. doi: 10.1177/0956797609356283

Berti, A., \& Frassinetti, F. (2000). When far becomes near: Re-maping of space by tool use. Journal of Cognitive Neuroscience12(415-420).

Bian, Z., \& Andersen, G. J. (2013). Aging and the perception of egocentric distance. Psychology and Aging, 28(3), 813-825.

Carter, L. F., \& Schooler, K. (1949). Value, need, and other factors in perception. Psychological Review, 56(4), 200-207.

Cole, S., Balcetis, E., \& Dunning, D. (2013). Affective signals of threat produce perceived proximity. Psychological Science, 24, 34-40.

Datta, D., Selvarajah, K., \& Davey, N. (2004). Functional outcome of patients with proximal upper limb deficiency-acquired and congenital. Clinical Rehabilitation, 18(2), 172-177.

Dudkiewicz, I., Gabrielov, R., Seiv-Ner, I., Zelig, G., \& Heim, M. (2004). Evaluation of prosthetic usage in upper limb amputees. Disability and Rehabilitation, 26(1), 60-63.

Durgin, F. H., Baird, J. A., Greenburg, M., Russell, R., Shaughnessy, K., \& Waymouth, S. (2009). Who is being deceived? The experimental demands of wearing a backpack. Psychonomic Bulletin \& Review, 16(5), 964-969. doi: 10.3758/PBR.16.5.964

Eves, F. F. (2016). Public health doesn't care what Fodor thinks. Journal of Applied Research in Memory and Cognition.

Eves, F. F., Thorpe, S. K. S., Lewis, A., \& Taylor-Covill, G. A. H. (2014). Does perceived steepness deter stair climbing when an alternative is available? Psychonomic Bulletin \& Review, 21(3), 637-644.

Firestone, C., \& Scholl, B. J. (in press). Cognition does not affect perception: Evaluating the evidence for 'top-down' effects. Behavioral and Brain Sciences.

Gibson, J. J. (1979). The Ecological Approach to Visual Perception. Boston: Houghton Mifflin.

Gray, R. (2013). Being selective at the plate: Processing dependence between perceptual variables relates to hitting goals and performance. Journal of Experimental Psychology: Human Perception and Performance, 39(4), 1124-1142.

Gray, R. (2016). Better understanding of the link between embodied perception and behavior needed before we can apply it. Journal of Applied Research in Memory and Cognition.

Gray, R., Navia, J. A., \& Allsop, J. (2014). Action-specific effects in aviation: What determines judged runway size? . Perception, 43, 145-154.

Lee, Y., Lee, S., Carello, C., \& Turvey, M. T. (2012). An archer's perceived form scales the "hitableness" of archery targets. Journal of Experimental Psychology: Human Perception and Performance, 38(5), $1125-1131$.

Leyrer, M., Linkenauger, S. A., Bulthoff, H. H., \& Mohler, B. J. (2015). The importance of postural cues for determining eye height in immersive virtual reality. PLOS ONE, 10(5), 1-23.

Lindner, H. Y., Nätterlund, B. S., \& Hermansson, L. M. N. (2010). Upper limb prosthetic outcome measures: review and content comparison based on International Classification of Functioning, Disability and Health. Prosthetics and Orthotics International, 34(2), 109-128.

Linkenauger, S. A., Bulthoff, H. H., \& Mohler, B. J. (2015). Virtual arm's reach influences perceived distance but only after experience reaching. Neuropsychologia, 70, 393-401. doi:

10.1016/j.neuropsychologia.2014.10.034 
Linkenauger, S. A., Geuss, M. N., Stefanucci, J. K., Leyrer, M., Richardson, B. H., Proffitt, D. R., ... Mohler, B. J. (2014). Evidence for hand-size constancy: The dominant hand as a natural perceptual metric. Psychological Science, 25(11), 2086-2094.

Linkenauger, S. A., Leyrer, M., Buelthoff, H. H., \& Mohler, B. J. (2013). Welcome to wonderland: The influence of the size and shape of a virtual hand on the perceived size and shape of virtual objects. PLOS ONE, 8(7), e68594.

Linkenauger, S. A., Mohler, B. J., \& Proffitt, D. R. (2011). Body-based perceptual rescaling revealed through the size-weight illusion. Perception, 40(10), 1251-1253. doi: Doi 10.1068/P7049

Linkenauger, S. A., Ramenzoni, V., \& Proffitt, D. R. (2010). Illusory shrinkage and growth: body-based rescaling affects the perception of size. Psychological Science, 21(9), 1318-1325. doi: 10.1177/0956797610380700

Linkenauger, S. A., Witt, J. K., Bakdash, J. Z., Stefanucci, J. K., \& Proffitt, D. R. (2009). Asymmetrical body perception: A possible role for neural body representations. Psychological Science, 20(11), 13731380.

Loomis, J. M. (2016). Proposed applications of research on action-specific effects are premature. Journal of Applied Research in Memory and Cognition.

Moeller, B., Zoppke, H., \& Frings, C. (in press). What a car does to your perception: Distance evaluations differ from within and outside a car. Psychonomic Bulletin \& Review.

Philbeck, J. W., \& Witt, J. K. (2015). Action-specific influences on perception and post-perceptual processes: Present controversies and future directions. Psychological Bulletin, 141(6), 11201144.

Proffitt, D. R., \& Linkenauger, S. A. (2013). Perception viewed as a phenotypic expression. In W. Prinz, M. Beisert \& A. Herwig (Eds.), Action Science: Foundations of an Emerging Discipline (pp. 171-198). Cambridge, MA: MIT Press.

Roeschlein, R. A., \& Domholdt, E. (1989). Factors related to successful upper extremity prosthetic use. Prosthetics and Orthotics International, 13(1), 14-18.

Sedgwick, H. (1986). Space perception. In K. R. Boff, L. Kaufman \& J. P. Thomas (Eds.), Handbook of Perception and Human Performance (Vol. 1, pp. 1-57). New York: Wiley.

Singh, M., \& Hoffman, D. D. (2013). Natural selection and shape perception. In S. J. Dickinson \& Z. Pizlo (Eds.), Shape Perception in Human and Computer Vision: An Interdisciplinary Perspective (pp. 171-185). London: Springer.

Stefanucci, J. K., \& Proffitt, D. R. (2009). The roles of altitude and fear in the perception of height. Journal of Experimental Psychology: Human Perception and Performance, 35(2), 424-438. doi: 10.1037/a0013894

Stefanucci, J. K., Proffitt, D. R., Banton, T., \& Epstein, W. (2005). Distances appear different on hills. Perception \& Psychophysics, 67(6), 1052-1060.

Sugovic, M., Turk, P., \& Witt, J. K. (in press). Perceived distance and obesity: It's what you weigh, not what you think. Acta Psychologica.

Sugovic, M., \& Witt, J. K. (2013). An older view on distance perception: older adults perceive walkable extents as farther. Experimental Brain Research. doi: 10.1007/s00221-013-3447-y

Taylor-Covill, G. A. H., \& Eves, F. F. (in press). Carrying a Biological "Backpack": Quasi-Experimental Effects of Weight Status and Body Fat Change on Perceived Steepness. Journal of Experimental Psychology: Human Perception and Performance.

Tenhundfeld, N. T., \& Witt, J. K. (in preparation). Substantiating a perceptual account: Evidence that distances on hills look farther than distances on flat ground.

Van der Hoort, B., \& Ehrsson, H. H. (2014). Body ownership affects visual perception of object size by rescaling the visual representation of external space. Attention, Perception, \& Psychophysics, 76(5), 1414-1428. 
Warren, W. H. (1984). Perceiving affordances: The visual guidance of stair climbing. Journal of Experimental Psychology: Human Perception and Performance, 10, 683-703.

Witt, J. K. (2011). Tool use influences perceived shape and perceived parallelism, which serve as indirect measures of perceived distance. Journal of Experimental Psychology: Human Perception and Performance, 37(4), 1148-1156. doi: 10.1037/a0021933

Witt, J. K. (2015). Awareness is not a necessary characteristic of a perceptual effect: Commentary on Firestone (2013). Perspectives on Psychological Science, 10(6), 865-872.

Witt, J. K., Linkenauger, S. A., Bakdash, J. Z., Augustyn, J. S., Cook, A., \& Proffitt, D. R. (2009). The long road of pain: chronic pain increases perceived distance. Experimental Brain Research, 192(1), 145-148. doi: 10.1007/s00221-008-1594-3

Witt, J. K., Linkenauger, S. A., \& Wickens, C. D. (2016). Action-specific effects in perception and their potential applications. Journal of Applied Research in Memory and Cognition.

Witt, J. K., \& Proffitt, D. R. (2005). See the ball, hit the ball - Apparent ball size is correlated with batting average. Psychological Science, 16(12), 937-938. doi: DOI 10.1111/j.1467-9280.2005.01640.x

Witt, J. K., Proffitt, D. R., \& Epstein, W. (2010). When and how are spatial perceptions scaled? Journal of Experimental Psychology: Human Perception and Performance, 36(5), 1153-1160. doi: Doi 10.1037/A0019947

Witt, J. K., \& Riley, M. A. (2014). Discovering your inner Gibson: Reconciling action-specific and ecological approaches to perception-action. Psychonomic Bulletin \& Review, 21(6), 1353-1370.

Witt, J. K., \& Sugovic, M. (2012). Does ease to block a ball affect perceived ball speed? Examination of alternative hypotheses. Journal of Experimental Psychology: Human Perception and Performance, 38(5), 1202-1214. doi: 10.1037/a0026512

Witt, J. K., \& Sugovic, M. (2013a). Catching ease influences perceived speed: Evidence for action-specific effects from action-based measures. Psychonomic Bulletin \& Review, 20, 1364-1370.

Witt, J. K., \& Sugovic, M. (2013b). Spiders appear to move faster than non-threatening objects regardless of one's ability to block them. Acta Psychologica, 143, 284-291.

Witt, J. K., Sugovic, M., \& Dodd, M. D. (in press). Action-specific perception of speed is independent of attention. Attention, Perception, \& Psychophysics.

Witt, J. K., Sugovic, M., Tenhundfeld, N. T., \& King, Z. R. (in press). An Action-Specific Effect on Perception that Avoids All Pitfalls. Behavioral and Brain Sciences.

Wright, F. V., Hubbard, S., Jutai, J., \& Naumann, S. (2001). The Prosthetic Upper Extremity Functional Index: development and reliability testing of a new functional status questionnaire for children who use upper extremity prostheses. Journal of Hand Therapy, 14(2), 91-104. 